\title{
Myosin II Activation and Actin Reorganization Regulate the Mode of Quantal Exocytosis in Mouse Adrenal Chromaffin Cells
}

\author{
Bryan W. Doreian, Tiberiu G. Fulop, and Corey B. Smith \\ Department of Physiology and Biophysics, Case Western Reserve University, Cleveland, Ohio 44106-4970
}

\begin{abstract}
Chromaffin cells of the adrenal medulla are innervated by the sympathetic nervous system. Stimulation causes chromaffin cells to fire action potentials, leading to the exocytosis of various classes of transmitters into the circulation. Low-frequency electrical stimulation (action potentials delivered at $0.5 \mathrm{~Hz}$ ) causes adrenal chromaffin cells to selectively release catecholamines through a kiss-and-run fusion event. Elevated electrical stimulation (action potentials at $15 \mathrm{~Hz}$ ) evokes fusion pore dilation, full granule collapse, and additional release of the neuropeptide-containing proteinaceous granule core. Here we apply single-cell electrophysiological, electrochemical, and fluorescence measurements to investigate the cellular mechanism for this shift in exocytic behavior. We show that at low-frequency stimulation, a filamentous-actin cell cortex plays a key role in stabilizing the kiss-and-run fusion event. Increased stimulation disrupts the actin cortex, driving full granule collapse. We show that pharmacological perturbation of the actin cortex supersedes stimulus frequency in controlling exocytic mode. Finally, we show that nonmuscle myosin II activation contributes to the cytoskeleton-dependent control of the fusion event. Inhibition of myosin II or myosin light chain kinase under elevated stimulation frequencies inhibits fusion pore dilation and maintains the granule in a kiss-and-run mode of exocytosis. These results demonstrate an essential role for activity-evoked cytoskeletal rearrangement and the action of myosin II in the regulation of catecholamine and neuropeptide exocytosis and represent an essential element of the sympathetic stress response.
\end{abstract}

Key words: actin; myosin II; exocytosis; chromaffin; neurotransmitter; stress

\section{Introduction}

Chromaffin cells of the adrenal medulla are a major output of the sympathetic nervous system and are innervated by the splanchnic nerve through a cholinergic synapse. Under basal sympathetic firing, chromaffin cells selectively release freely soluble catecholamines through an $\Omega$-form kiss-and-run fusion event (Elhamdani et al., 2006; Fulop and Smith, 2006). This exocytic mode is characterized by transient fusion of the granule with the cell membrane, maintenance of basic granule morphology, and retention of the dense proteinaceous granule core (Fulop et al., 2005). In contrast, under acute stress, increased splanchnic firing and subsequent acetylcholine release drive chromaffin cells to increase their activity level (Brandt et al., 1976; Kidokoro and Ritchie, 1980), resulting in elevated catecholamine output. Under these conditions, the restricted fusion pore dilates, leading to a full granule collapse and expulsion of both catecholamine and the neuropeptide-containing proteinaceous core (Rahamimoff

Received Jan. 2, 2008; revised March 21, 2008; accepted March 24, 2008.

This work was supported by National Science Foundation Grant IBN-0344768 and National Institutes of Health Grants 1R01NS052123 (C.B.S.), T32 HL 07887 (B.W.D.), and T32 HL 07653 (T.G.F.). We thank Dr. Shyue-An Chan for expert help in design and execution of the experiments in this study as well as critical comment on this manuscript. We would also like to thank Katherine Trueblood-Doreian for critical comments.

Correspondence should be addressed to Dr. Corey Smith, Department of Physiology and Biophysics, Case Western Reserve University, Cleveland, OH 44106-4970. E-mail: corey.smith@case.edu.

D01:10.1523/JNEUROSCI.0008-08.2008

Copyright $\odot 2008$ Society for Neuroscience $\quad$ 0270-6474/08/284470-09\$15.00/0 and Fernandez, 1997; Fulop et al., 2005). Thus, dependent on stimulation intensity, chromaffin cells use two modes of exocytosis that differentially release catecholamine and neuropeptide transmitter molecules. The cellular mechanism and regulation of the transition from kiss-and-run to full granule collapse is not fully understood. Improper regulation of the acute stress response can cause pathological conditions such as hypertension, diabetes, and depression (Habib et al., 2001).

Many studies have focused on the role played by the cytoskeleton in the control of catecholamine release. In resting conditions, chromaffin cells exhibit a subplasmalemmal filamentous actin (F-actin) cortical network that is thought to act as a physical barrier to granule recruitment to the plasma membrane and limit secretory function (Vitale et al., 1995; Barclay et al., 2003). Stimulation leads to a partial dissolution of the F-actin cortex, allowing for granule recruitment to the cell periphery for sustained transmitter release (Vitale et al., 1991). It has also been shown that nonmuscle myosin II, associated with the F-actin network, may play a role in active granule recruitment (Neco et al., 2002). On the single-granule level, inhibition of myosin II decreases the catecholamine quantal size of single fusion events (Neco et al., 2004). However, mechanistic description for how the actin/myosin II system regulates quantal size remains to be determined.

The goal of this study was to investigate the role of activitymediated actin reorganization and myosin II function in the control of quantal exocytosis. We used carbon-fiber amperometry, 
capacitance noise analysis, fluorescence microscopy, and peptide transfection techniques to investigate the mode of exocytosis. We determined the role of the actin cytoskeleton and myosin II in regulating the activity-dependent transition from kiss-and-run to full-collapse exocytosis. Our data demonstrate that the characteristic $\Omega$-form kiss-and-run exocytosis is stabilized by an intact F-actin cortex. Elevated stimulation resulted in a cooperative partial disruption of the F-actin cortex and activation of myosin II to drive collapse of the $\Omega$-figure to achieve the full-collapse exocytic mode. Thus, we have identified a role for F-actin and myosin II in the control and regulation of a proper sympathetic stress response by controlling the mode of exocytosis at the single-granule level.

\section{Materials and Methods}

Chromaffin cells isolated from the adrenal medullae of adult C57BL/6 mice (The Jackson Laboratory, Bar Harbor, ME; 8 weeks old) were used in this study. All anesthesia and euthanasia protocols were reviewed and approved by the institutional animal care and use committee of Case Western Reserve University, an accredited oversight body (federal animal welfare assurance \#A3145-01). Animals were deeply anesthetized by isoflurane inhalation and killed by decapitation. All chemicals and reagents were obtained from Sigma (St. Louis, MO) unless otherwise noted in the text.

Cell preparation. Adrenal glands were removed immediately after animals were killed, and placed in an ice-cold dissociation solution containing (in mM) $80 \mathrm{Na}$-glutamate, $55 \mathrm{NaCl}, 6 \mathrm{KCl}, 1 \mathrm{MgCl}_{2}, 10 \mathrm{HEPES}$, and 10 glucose, $\mathrm{pH} 7.0$, and osmolarity was adjusted to $280 \mathrm{mOsm}$. Glands were trimmed of fat, and the adrenal cortex was dissected from the medulla. Cells were isolated as previously described (Fulop et al., 2005) and plated on 25-mm-diameter round cover glasses and cultured in $\sim 2 \mathrm{ml}$ of DMEM growth medium supplemented with ITS-X artificial serum substitute ( $1 \times$; Invitrogen, Carlsbad CA) and penicillin/streptomycin (20 $\mathrm{U} / \mathrm{ml}$ each). The cells were incubated at $35^{\circ} \mathrm{C}$ in $10 \% \mathrm{CO}_{2}$, and the experiments were performed at $\sim 25^{\circ} \mathrm{C} 2-3 \mathrm{~d}$ after cell preparation.

Electrophysiology and electrochemistry. All electrophysiological recordings were performed in the perforated-patch configuration (Korn and Horn, 1989) as previously described (Chan and Smith, 2001), with some modifications described here. The electrical stimulus protocols were delivered by either an EPC-9 or an EPC-10 amplifier (HEKA Elektronik, Lambrecht, Germany) controlled by Pulse (HEKA Elektronik; version 8.8). Cell capacitance was measured by imposing a $635 \mathrm{~Hz}$ sine wave 25 $\mathrm{mV}$ in amplitude on a holding potential of $-80 \mathrm{mV}$. Cell capacitance and conductance were determined by the sine + DC method (Gillis, 1995) implemented through the built-in lock-in module in Pulse. Cells were stimulated with action potential equivalent $(\mathrm{APe})$ waveforms embedded in the sine wave holding potential at either 0.5 or $15 \mathrm{~Hz}$. The APe was formed from ramp segments as follows [start potential (in $\mathrm{mV}$ ), end potential (in $\mathrm{mV}$ ), duration (in $\mathrm{ms}$ )]: $-80,50,2.5 ; 50,-90,2.5 ;-90$, $-80,2.5$. Voltage-dependent sodium and calcium influx, elicited catecholamine release, and capacitance jumps in response to APe stimulation have been shown to be statistically identical to native AP waveforms (Chan and Smith, 2001). The internal pipette solution contained the following composition (in $\mathrm{mm}$ ): 135 Cs-glutamate, 10 HEPES-H, 9.5 $\mathrm{NaCl}, 0.5 \mathrm{TEA}-\mathrm{Cl}$, and 0.53 amphotericin $\mathrm{B}$. During all recordings, the cells were constantly superfused at a rate of $\sim 1 \mathrm{ml} / \mathrm{min}$ with a Ringer's solution of the following composition (in $\mathrm{mM}$ ): $150 \mathrm{NaCl}, 10$ HEPES-H, 10 glucose, $2.8 \mathrm{CaCl}_{2}, 2.8 \mathrm{KCl}$, and $2 \mathrm{MgCl}_{2}$. The osmolarity was adjusted to $320 \mathrm{mOsm}$ with mannitol, and the $\mathrm{pH}$ was adjusted to 7.2. The junction potential for this internal/Ringer's solution set was measured to be approximately $-13 \mathrm{mV}$, and all potentials were adjusted accordingly. For elevated potassium stimulation, $\mathrm{KCl}$ was increased to either 8 or 30 $\mathrm{mm}$, and $\mathrm{NaCl}$ was reduced to maintain osmolarity (Fulop and Smith, 2007). Cell capacitance-noise-analysis detection of $\Omega$-form kiss-and-run granule fusion was performed as described previously (Fulop and Smith, 2006).

Amperometric recordings were also performed as described previ- ously (Fulop et al., 2005). Commercially available $5 \mu \mathrm{m}$ diameter carbon fiber electrodes (ALA Scientific, Westbury, NY) were used for catecholamine detection. The carbon fiber tip was cut before each recording, and the fiber was held at $+650 \mathrm{mV}$. The fiber tip was positioned close to the cell membrane to minimize diffusion distance from the cell membrane. Amperometric current was recorded using a dedicated amplifier (VA10x; ALA Scientific) with a modified head stage containing a $1 \mathrm{G} \Omega$ feedback resistor to minimize noise. Recorded signals were passed through a four-pole analog Bessel filter at a cutoff frequency of $1.3 \mathrm{kHz}$ and sampled at $20 \mathrm{kHz}$ through an ITC-1600 (Instrutech, Port Washington, NY) into IGOR Pro (WaveMetrics, Lake Oswego, OR).

Pharmacological agents. Cells were treated with $2 \mu \mathrm{M}$ cytochalasin D (Sigma), $2 \mu \mathrm{M}$ jasplakinolide (Invitrogen), $10 \mu \mathrm{M}$ ML7 (EMD Biosciences, San Diego, CA), $2 \mu$ M MLCK inhibitor peptide 18 (M18; EMD Biosciences), or $25 \mu \mathrm{m}$ blebbistatin (Biomol, Plymouth Meeting, PA) as indicated in the text. All preincubations were performed by addition of the agent to the culture medium and incubation at $35^{\circ} \mathrm{C}$ to facilitate reagent uptake. This preincubation was $10 \mathrm{~min}$ in duration for all reagents except the cell-permeant M18 MLCK inhibitory peptide. Because of its larger size, the M18 peptide was preincubated in the culture medium for $20 \mathrm{~min}$. After pretreatment, cells were immediately transferred to the recording chamber, superfused with the appropriate reagentcontaining recording Ringer's solution, and used for experimentation.

Imaging and fluorometry. Fluorescence images were acquired on an Olympus (Tokyo, Japan) IX-81 inverted microscope with a $100 \times$ oilimmersion objective (numerical aperture, 1.3). Excitation illumination was provided by a TILL Polychrometer IV (TILL Photonics, Pleasanton, CA) under the control of SlideBook image acquisition software (SlideBook 4.1; Intelligent Imaging, Denver, CO). Images were collected with a cooled CCD camera (Retiga EXi; QImaging, Burnaby, British Columbia, Canada) at a set exposure time of $5 \mathrm{~ms}$ to standardize image intensity and to minimize photobleach during the illumination. Dextran staining and quantification were performed as previously described (Fulop et al., 2005). Actin labeling and quantification of F-actin were performed as follows: cells were pretreated with $2 \mu \mathrm{M}$ cytochalasin D or $2 \mu \mathrm{M}$ jasplakinolide by bath application. Cells were then patch clamped and stimulated at 0.5 or $15 \mathrm{~Hz}$ (as indicated in text) or with external potassium matched to provide identical stimulation intensity and secretory behavior (Fulop and Smith, 2007). Immediately after stimulation, cells were fixed at the pipette tip by bath application of $4 \%$ paraformaldehyde for 25 min (Fulop et al., 2005). After being washed three times with $1 \times$ PBS, cells were bathed in $1 \times \mathrm{PBS}$ for $5 \mathrm{~min}$. At this point, the patch pipette was gently lifted from the cell. Next, $1.5 \mathrm{ml}$ of $0.15 \%$ Triton X-100 was superfused into the dish and left for $30 \mathrm{~min}$ to permeabilize the cell membrane. Triton X-100 was then removed by three washes in $1 \times$ PBS, and cells were incubated with $5 \mu \mathrm{l}(200 \mathrm{U} / 1.5 \mathrm{ml}$; Invitrogen $)$ rhodamineconjugated phalloidin in $1.5 \mathrm{ml}$ of $1 \times$ PBS. Cells were then imaged as above.

Data analysis. Image and data analysis of fluorescence images were performed using custom-written macros in IGOR Pro (WaveMetrics). Single amperometric spikes were analyzed in IGOR Pro with a macro modified from the original "Spike" package (Gomez et al., 2002). Nonparametric Mann-Whitney statistical analysis of medians was performed using Minitab (version 15; Minitab, State College, PA). Statistical significance was tested at $99.99 \%(p<0.001)$ confidence level. Statistical significance for each category plot of each mean parameter was determined by Student's $t$ test. Statistical significance was tested at $95 \%(p<$ $0.05)$ confidence level. Data are expressed as mean \pm SEM.

\section{Results}

Recent work has demonstrated a mechanistic shift in the mode of exocytosis under different stimulation conditions in chromaffin as well as other secretory cell types (Aravanis et al., 2003; Fulop et al., 2005; Richards et al., 2005; Elhamdani et al., 2006). Work from our group and others has shown that chromaffin cells exhibit a rapid and transient kiss-and-run mode of exocytosis when challenged with modest stimulation conditions. Increased electrical or chemical stimulation shifts the mode of exocytosis to a 
more complete full-collapse secretory mode. We initiated a series of experiments designed to test whether activity-dependent myosin II activation and reorganization of the actin cortex play roles in this shift in exocytic mechanism. We used pharmacological tools to manipulate the balance between depolymerized and polymerized actin, as well as the function of myosin light chain kinase (MLCK) and myosin II. To quantify their effect, we assayed granule fusion, catecholamine release, and subsequent membrane trafficking under low-frequency and high-frequency action potential stimulation, which elicit kiss-and-run and fullcollapse exocytosis, respectively.

\section{Disrupting the actin cortex increases amperometric spike amplitude}

Carbon fiber amperometry is a high-resolution method for recording the kinetics of catecholamine release from single secretory granules (Wightman et al., 1991; Chow et al., 1992). This technique is capable of resolving the kinetics of both the initial opening and dilation of the granule-plasmalemma fusion pore as well as total quantal size of the fusion event (Alvarez de Toledo et al., 1993; Albillos et al., 1997; Artalejo et al., 2002). Increasing cell stimulation has been shown to increase quantal size (Elhamdani et al., 2001) as well as the rate of catecholamine release through an activity-mediated shift from kiss-and-run to full granule collapse (Fulop and Smith, 2006). We used amperometry to quantify the kinetics of catecholamine release from cells stimulated with APe voltage templates delivered at 0.5 or $15 \mathrm{~Hz}$ in perforated-patch voltage clamp. These conditions mimic splanchnic input under sympathetic tone or acute stress, respectively (Kidokoro and Ritchie, 1980; Chan and Smith, 2001; Wilson et al., 2002).

Representative amperometric recordings from single cells stimulated with either 0.5 or $15 \mathrm{~Hz}$ APe trains are shown (Fig. 1a). From such experiments, we analyzed single-spike parameters (Fig. 1bi) that correlate to fusion pore dilation and thus overall exocytic mode. Spike amplitude and spike charge (an index of total catecholamine content) have been shown to increase with dilation of the fusion pore (Alvarez de Toledo et al., 1993; Albillos et al., 1997; Lindau and Alvarez de Toledo, 2003; Fulop and Smith, 2006). An initial analysis of these parameters showed that they followed, not a normal, but rather a skewed distribution (Neco et al., 2004). Cumulative probability plots for each parameter are provided (Fig. 1bii). Insets in each subpanel show boxand-whisker plots for each distribution. Nonparametric MannWhitney median tests indicate that both parameters, spike amplitude and spike charge, are significantly smaller under 0.5 $\mathrm{Hz}$ versus $15 \mathrm{~Hz}$ APe stimulation. We also analyzed population means that also showed statistical significance in each parameter (supplemental table S1, available at www.jneurosci.org as supplemental material). Thus, by either median or more conventional mean-based statistical analyses, catecholamine secretion kinetics evoked by $15 \mathrm{~Hz}$ electrical APe stimulation are more rapid than those under $0.5 \mathrm{~Hz}$ stimulation and indicate a dilated fusion pore under higher stimulation intensity.

Previous studies have shown that the distinct actin cortex normally observed in chromaffin cells is disrupted by cell stimulation (Vitale et al., 1995; Trifaró et al., 2000; Giner et al., 2007). We set out to test whether pharmacological perturbation of the actin cortex could mimic the difference in stimulation intensity by regulating the kinetics of catecholamine secretion. Cells were pretreated (see Materials and Methods) with pharmacological agents that either disrupt or stabilize the actin cortex $(2 \mu \mathrm{M}$ cytochalasin D or $2 \mu \mathrm{M}$ jasplakinolide, respectively) and stimulated at 0.5 or 15 Hz. The actin cortex was then visualized with rhodamine-
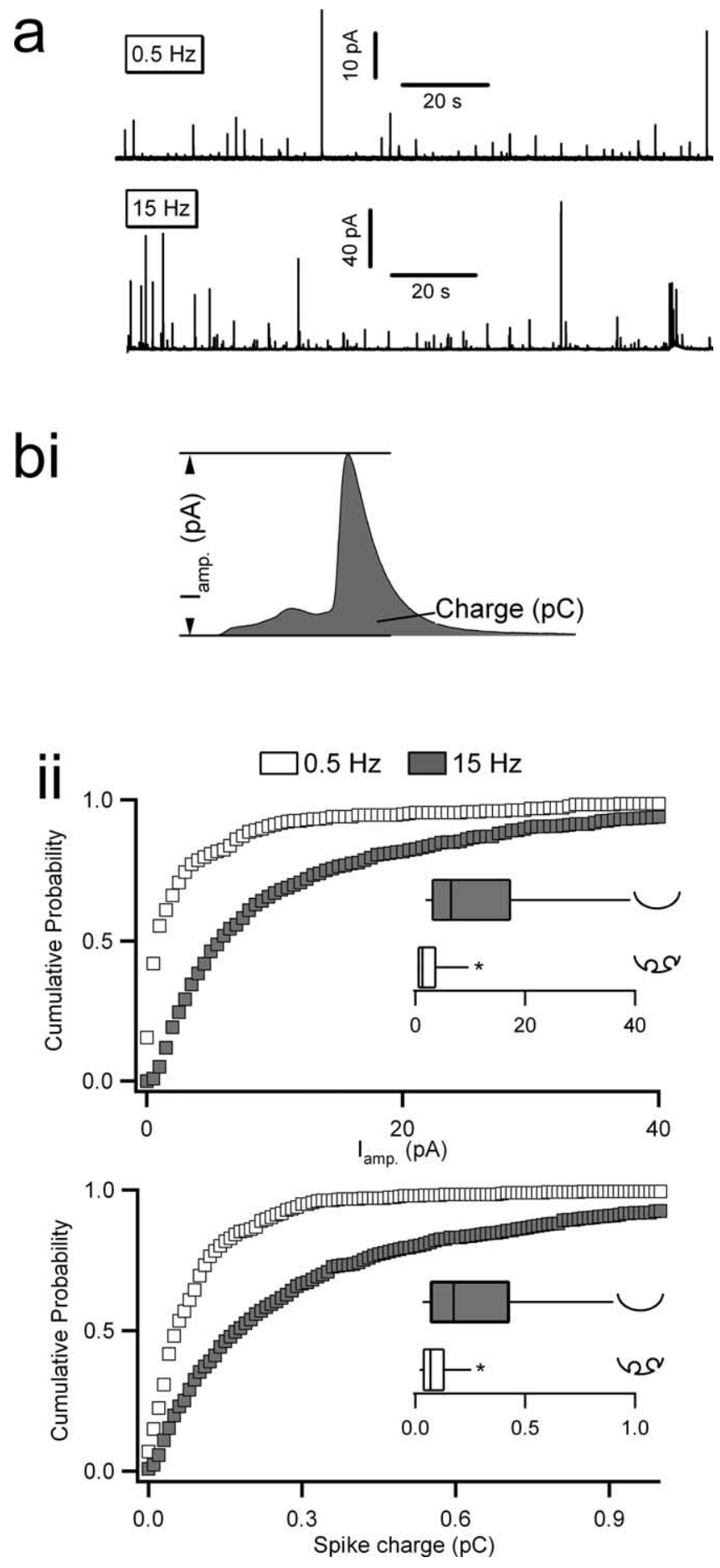

Figure 1. Amperometric analysis of catecholamine release under 0.5 versus $15 \mathrm{~Hz}$ electrical stimulation. Chromaffin cells were held at $-80 \mathrm{mV}$ in the perforated-patch configuration and stimulated with action potential equivalent waveforms. $\boldsymbol{a}$, Representative traces from cells continuously stimulated with action potential equivalent voltage templates at 0.5 or $15 \mathrm{~Hz}$ are provided (note the difference in scale). Equivalent time windows are shown for better comparison. Individual spikes were identified using an automated detection algorithm and then manually confirmed. bi, Two kinetic parameters, maximum spike amplitude (in picoamperes) and total spike charge (in picocoulombs), were measured for each individual spike. bii, Pooled cumulative probability plots for spike amplitude (top) and charge (bottom) are provided. Insets show box-and-whisker plots for their respective datasets. Parameters for the box-and-whisker plots are as follows: smallest nonoutlier, first quartile, median, third quartile, and largest nonoutlier observation. Statistical significance for medians was determined by a Mann-Whitney nonparametric median analysis, and asterisks in each box-and-whisker plot indicate statistical significance ( $p<0.001)$. Icons next to each box-and-whisker category provide interpretation of the mode of fusion. $n=12$ cells from 7 preparations for 0.5 and 10 cells from 7 preparations for $15 \mathrm{~Hz}$. 

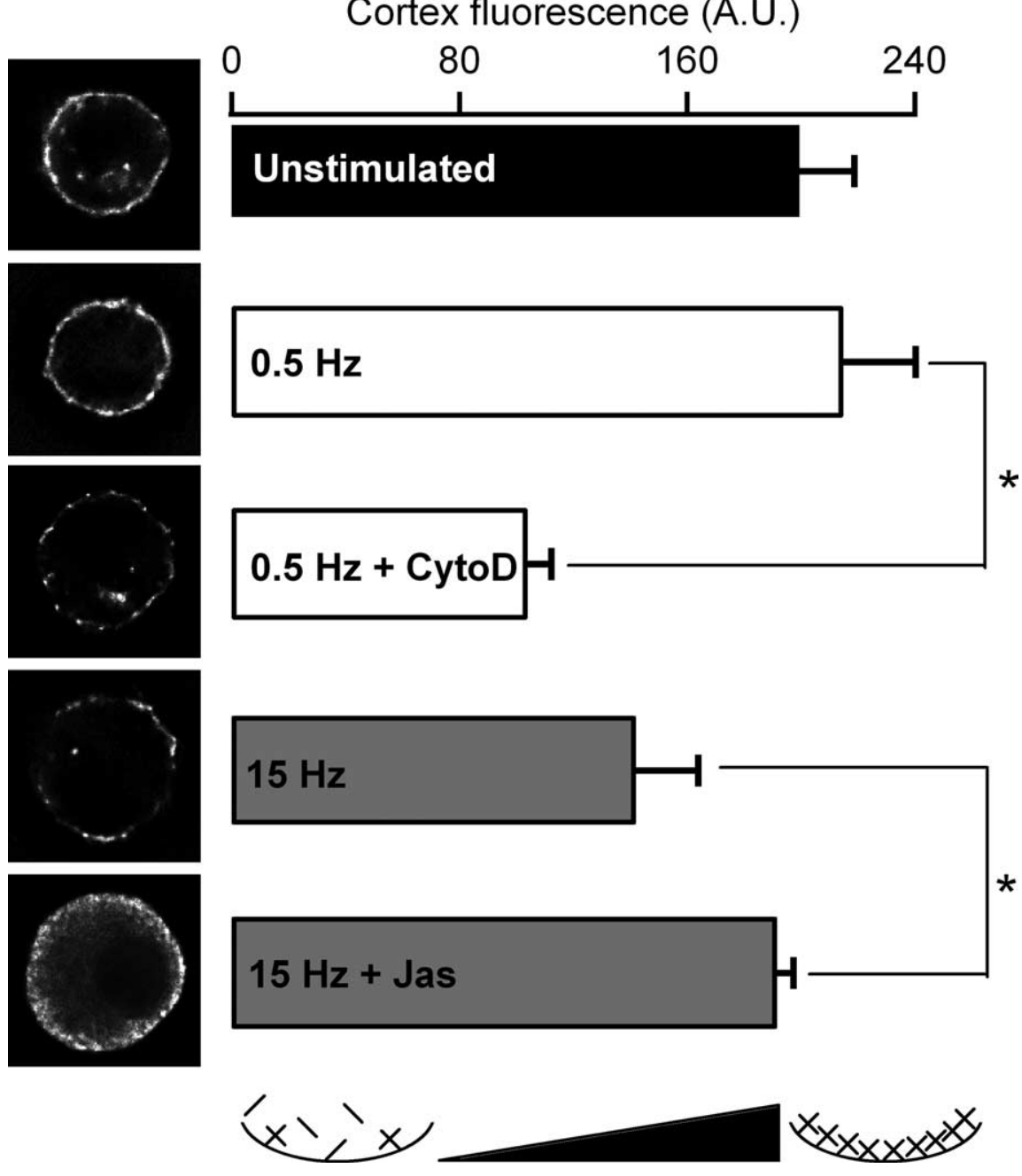

Figure 2. Rhodamine-phalloidin imaging of the actin cortex. Chromaffin cells were stimulated as in Figure 1, but immediately fixed after stimulation by perfusion with a paraformaldehyde-containing Ringer's solution. After fixation, cells were permeabilized by Triton X-100 perfusion and stained with rhodamine-conjugated phalloidin to provide a fluorescence-based index of filamentous actin. Each cell was imaged as a stack with $0.25 \mu \mathrm{m} z$-steps. All cells in the dataset were imaged with identical illumination, exposure, and camera gain settings to enable quantitative cross-comparison of intensity levels. Equatorial images of representative cells for each condition are provided (left column), and quantified mean cortical staining (cell periphery and $1 \mu \mathrm{m}$ under periphery) is provided in the bar-graph format (right column). Abbreviations (ytoD and Jas represent cells treated with cytochalasin D and jasplakinolide, respectively, in this and other figures. An icon at the bottom of the right column represents the status of the cortical actin as a function of fluorescence intensity. $n=15$ cells from 3 preparations for all conditions. Statistical significance for each mean parameter was determined by Student's $t$ test, and an asterisk in each category indicates statistical significance with respect to control $(p<0.05)$. Error bars represent SEM.

conjugated phalloidin $(6 \mu \mathrm{M})$, a compound that binds to filamentous but not monomeric actin (Wieland, 1986). We took advantage of this polymerization-specific staining to estimate the relative disruption of the actin cortex under different stimulation conditions. Single-cell images (Fig. 2, left column) as well as quantified phalloidin staining (Fig. 2, right column) show that $0.5 \mathrm{~Hz}$ stimulation had no effect on the actin cortex when compared with unstimulated control cells. However, pretreatment with cytochalasin D significantly decreased the amount of polymerized F-actin at the cell periphery. Stimulation at $15 \mathrm{~Hz}$ resulted in a punctate phalloidin staining at the cell periphery and a decreased amount of cortical F-actin compared with unstimulated control cells. This stimulus-dependent cortical disruption was blocked by pretreatment with jasplakinolide under $15 \mathrm{~Hz}$ stimulation.

Next we tested the direct effects of cytochalasin D and jas- plakinolide on catecholamine release. We measured single amperometric spike amplitude and charge as previously (Fig. 1). Cells were pretreated with cytochalasin D as above and then stimulated with trains of APe waveforms at $0.5 \mathrm{~Hz}$ (Fig. 3ai). The cumulative probability plot for spike amplitude is presented along with paired control data for comparison (Fig. 3aii). The inset box-and-whisker plot indicates that the median spike amplitude was significantly enhanced with cytochalasin D treatment when compared with untreated control cells. Cytochalasin D also significantly increased spike charge. Furthermore, cytochalasin D treatment had no effect under $15 \mathrm{~Hz}$ stimulation, consistent with the already disrupted actin cortex observed at this activity level in Figure 2 (for complete dataset, see supplemental table S1, available at www.jneurosci.org as supplemental material). We then treated cells with jasplakinolide to stabilize the actin cortex, and stimulated with $15 \mathrm{~Hz}$ APe trains. A representative single recording is provided (Fig. 3bi). Single spikes were again quantified for each secretory event, and the cumulative probability plot for their amplitude and the corresponding box-andwhisker median analysis plot are provided (Fig. 3bii). These data show that actin stabilization with jasplakinolide acted to significantly decrease spike amplitude when compared with untreated cells stimulated in the same manner (Fig. 3bii). Again, a significant decrease was also observed for spike charge with jasplakinolide treatment under $15 \mathrm{~Hz}$ stimulation, whereas jasplakinolide had no effect on amperometric parameters acquired under $0.5 \mathrm{~Hz}$ stimulation (supplemental table S1, available at www.jneurosci.org as supplemental material). Thus, pharmacological manipulation of the actin cortex controls normal activity-specific secretory behavior. Whether achieved in an activitydependent manner or by pharmacological perturbation, disruption of F-actin favors large, rapid spikes associated with full granule collapse. An intact actin cortex, as present under light electrical stimulation or as a result of pharmacological stabilization even under elevated stimulation, favors smaller and slower spikes that are characteristic of kiss-and-run exocytosis (Fulop and Smith, 2006).

\section{Myosin II activity is required for the activity-dependent} increase in amperometric spike amplitude

Stabilizing networked actin partially, but not completely, converted the spike parameters measured under $15 \mathrm{~Hz}$ to those measured under $0.5 \mathrm{~Hz}$ stimulation. We considered other potential contributors that may regulate the activity-dependent and F-actin-dependent shift in exocytic mode. A number of studies have suggested that myosin II may play a role in regulating the exocytic mode. For example, it was shown that potassium stim- 

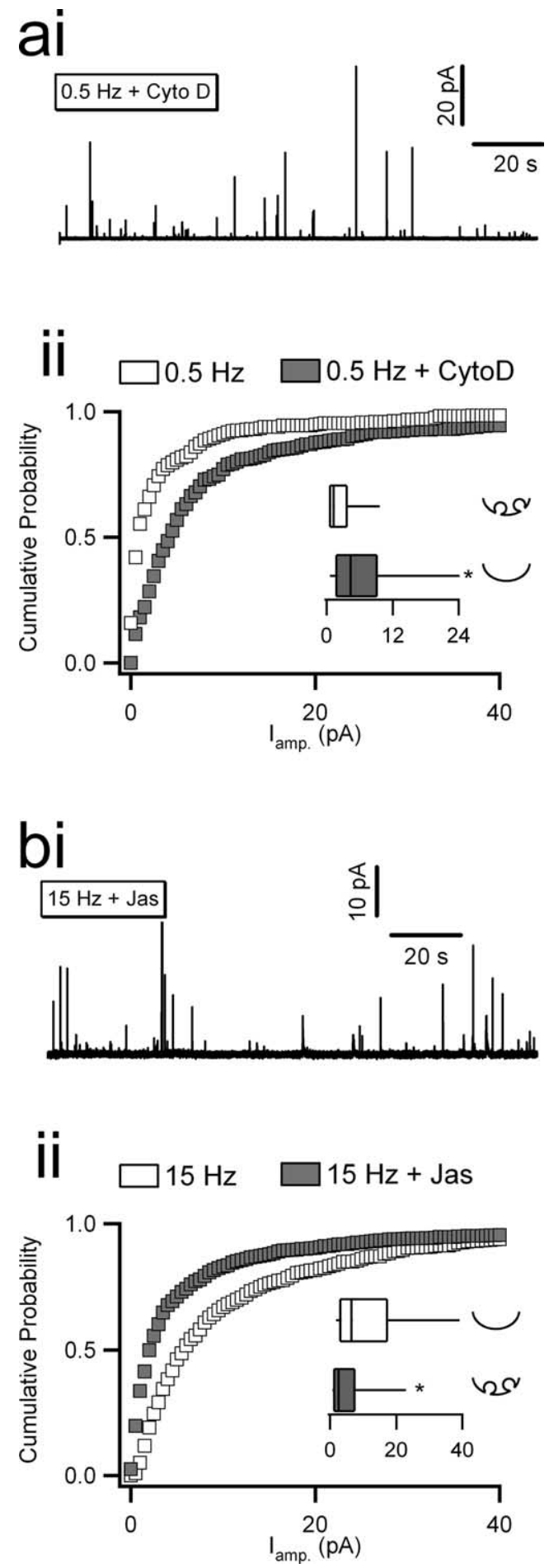

Figure 3. Actin-modifying reagents affect the kinetics of catecholamine release under $0.5 \mathrm{~Hz}$ stimulation. Chromaffin cells were voltage clamped and stimulated as in previous figures. Cells were pretreated with CytoD ( $2 \mu \mathrm{m}$ for $10 \mathrm{~min}$ ) and stimulated with trains of action potentials at $0.5 \mathrm{~Hz}$. ai, A sample raw record is plotted. Single amperometric spikes were analyzed as in Figure 1. aii, Cumulative probability plots for spike amplitude are provided for both control and CytoD datasets ( $n=10$ and 12 cells from 7 preparations for cytochalasin $D$ and control conditions, respectively). The inset box-and-whisker plot shows that CytoD treatment acted to increase the median spike amplitude compared with control $(p<0.001)$. To test the role of actin stabilization under elevated firing, cells were treated with Jas ( $2 \mu \mathrm{m}$ for $10 \mathrm{~min}$ ) and stimulated at $15 \mathrm{~Hz}$. $\boldsymbol{b i}$, A representative raw data record is provided. $\boldsymbol{b i i}$, Spike amplitude was measured for jasplakinolide-treated cells and is coplotted with control data collected from untreated cells stimulated at the same frequency. The inset box-and-whisker plot shows that jasplakinolide treatment acted to decrease the median spike amplitude compared with control $(p<0.001$; $n=10$ and 15 cells from 7 preparations for jasplakinolide and control conditions, respectively). ulation induces a calcium-dependent phosphorylation of the regulatory subunit of myosin light chain (Gutierrez et al., 1989), which serves to regulate the interaction of myosin with F-actin. Additionally, myosin II has been shown to be localized to the cell cortex, suggesting that its role is close to the plasma membrane (Rosé et al., 2002). Last, previous studies have shown that myosin II plays a role in the regulation of amperometric spike amplitude and total spike charge (Neco et al., 2004). Given these results, and that actin and myosin II represent a functional pairing, we investigated the role of myosin II activation and activity under 0.5 and $15 \mathrm{~Hz}$ APe stimulation.

We treated cells with either MLCK- or myosin II-specific inhibitors and analyzed amperometric events evoked under 0.5 and $15 \mathrm{~Hz}$ stimulation. Chromaffin cells were pretreated with either of the MLCK inhibitors ML7 (10 $\mu \mathrm{M})$ or M18 peptide $(2 \mu \mathrm{M})$. ML7 is a cell-permeant molecule that binds to the ATP-binding pocket of the enzyme, blocking MLCK-mediated phosphorylation of myosin, a necessary requisite to myosin motor function (Saitoh et al., 1987). M18 is a cell-permeant peptide fragment that competitively competes for binding to the substrate-binding pocket of the MLCK enzyme (Lukas et al., 1999; Neco et al., 2002). Additionally, we directly targeted myosin II by pretreatment with blebbistatin $(25 \mu \mathrm{M})$, a specific myosin II inhibitor that blocks ATP hydrolysis and thus motor activity. Chromaffin cells pretreated with the MLCK inhibitors ML7 or M18 showed a clear reduction in spike amplitude evoked under $15 \mathrm{~Hz}$ stimulation (Fig. 4a,b). ML7 and M18 decreased spike charge at $15 \mathrm{~Hz}$, but had no effect at $0.5 \mathrm{~Hz}$ (supplemental table S1, available at www.jneurosci.org as supplemental material). Direct targeting of myosin II by pretreatment with blebbistatin mirrored these results; spike amplitude was decreased at $15 \mathrm{~Hz}$ (Fig. 4c), with no effect at $0.5 \mathrm{~Hz}$ (supplemental table S1, available at www. jneurosci.org as supplemental material). Furthermore, a combined treatment with an actin stabilizer and a MLCK inhibitor, followed by stimulation at $15 \mathrm{~Hz}$, completely converted catecholamine release kinetics; spike amplitude was statistically identical to that measured from untreated cells stimulated at $0.5 \mathrm{~Hz}$ (Fig. 4d). A similar negative result was obtained for spike charge (supplemental table S1, available at www.jneurosci.org as supplemental material). Thus, in addition to the reorganization of the actin cortex, elevated cell firing leads to activation of myosin II through MLCK. This series of events contributes to the transition in exocytic mode for activity-dependent catecholamine release.

\section{Variance analysis indicates actin and myosin II regulate fusion pore dilation}

Cell capacitance measurements have long been used to measure granule fusion and provide an index of exocytosis in chromaffin cells (Neher and Marty, 1982). Recently, cell capacitance variance analysis has proven useful for detecting the accumulation of $\Omega$-figures during kiss-and-run exocytosis (Fulop and Smith, 2006). Briefly, during kiss-and-run exocytosis, the $\Omega$-form fusion intermediate adds an element to the normally simple equivalent circuit of a voltage-clamped chromaffin cell. In frequencydomain capacitance recordings, the additional circuit element includes the fusion pore conductance, which contributes to the Johnson noise of the circuit and increases the variance of the capacitance signal (Chen and Gillis, 2000). Thus, accumulation of $\Omega$-figures during kiss-and-run exocytosis increases capacitance variance by an amount proportional to the total number of fusion events. During activity-dependent transition to fullcollapse exocytosis, electrical conductance through the $\Omega$-figure fusion pore increases as it dilates to the point that the $\Omega$-figure 

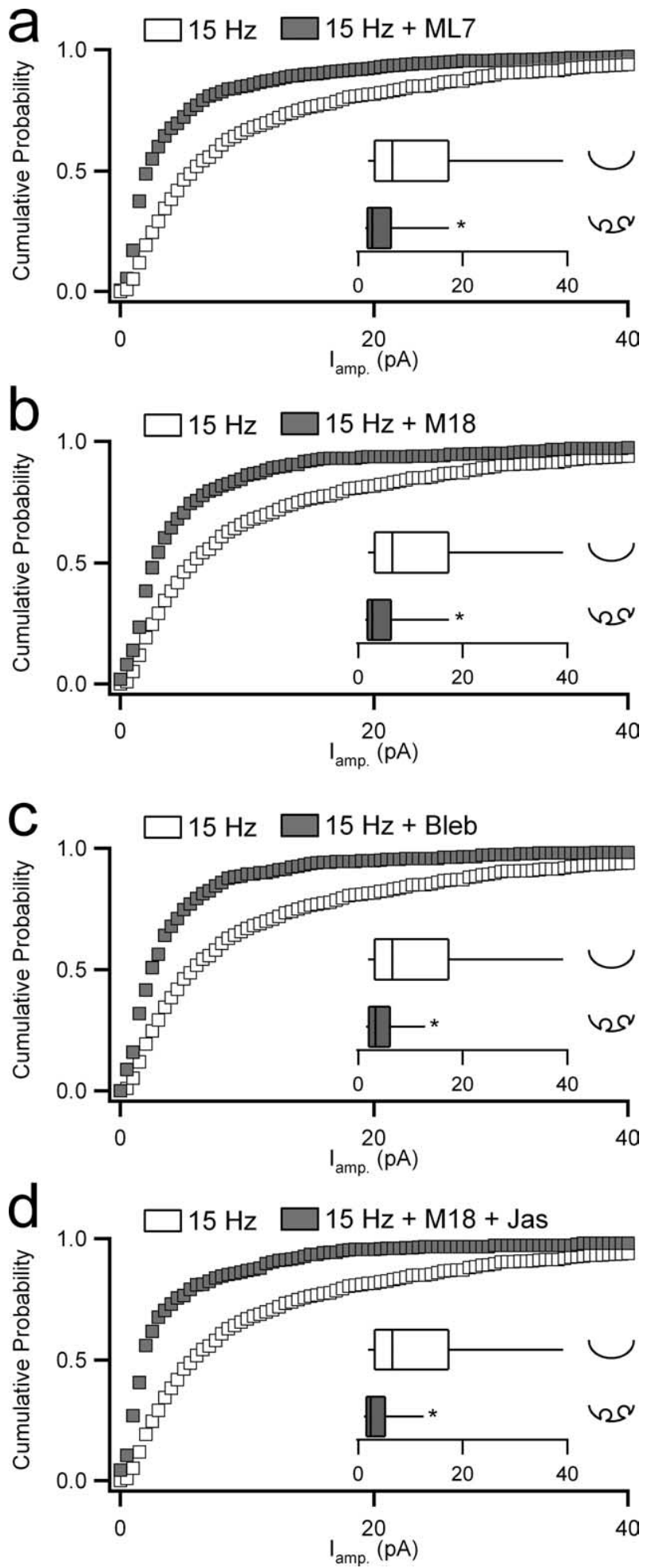

Figure 4. Myosin II-modifying reagents affect the kinetics of catecholamine release under $15 \mathrm{~Hz}$ stimulation. Chromaffin cells were voltage clamped and stimulated as in previous figures. $\boldsymbol{a}, \boldsymbol{b}$, Cells were pretreated with agents that block MLCK activity (10 $\mu \mathrm{m} \mathrm{ML7} \mathrm{in} \boldsymbol{a} ; n=11$ cells from 5 preparations; $2 \mu \mathrm{MM} 18$ in $\boldsymbol{b} ; n=8$ cells from 5 preparations) and stimulated with trains of action potentials at $15 \mathrm{~Hz}$. Single amperometric spikes were analyzed and plotted as in previous figures. The inset box-and-whisker plots show that disruption of normal MLCK activity decreased median spike amplitude compared with control cells ( $p$ values $<0.001$ ). c, Likewise, cells pretreated with blebbistatin (Bleb; $25 \mu \mathrm{m} ; n=8$ cells from 6 preparations), an agent that directly blocks myosin II activity, resulted in decreased spike amplitude under $15 \mathrm{~Hz}$ stimulation by median analysis ( $p$ values <0.001). $\boldsymbol{d}$, Cotreatment with M18 and Jas, to block MLCK and stabilize the actin cortex, resulted in less than an additive effect as determined by median analysis ( $p$ values $<0.001 ; n=10$ and 8 cells, respectively, from 6 preparations). becomes indistinguishable from the cell surface, removing its contribution to the total capacitance variance. In this manner, kiss-and-run exocytosis stably elevates capacitance variance, whereas full-collapse exocytosis does not (Fulop and Smith, 2006).

We took advantage of this technique to determine whether disrupting the actin cortex affected the accumulation of $\Omega$-figures normally observed in kiss-and-run exocytosis under $0.5 \mathrm{~Hz}$ stimulation. The standard deviation of the capacitance signal was measured in response to stimulation (Fig. $5 a, \sigma$ ). Variance is then determined simply as the square of the standard deviation $\left[\sigma^{2}\right.$ (in femtofarads squared)]. In addition to control recordings, chromaffin cells were again treated with either cytochalasin D or jasplakinolide as described above and stimulated at 0.5 or $15 \mathrm{~Hz}$. Data were pooled for each condition, and mean variance values are plotted (Fig. $5 b$ ). As expected, action potential stimulation at $0.5 \mathrm{~Hz}$ resulted in relatively high capacitance variance, indicating the accumulation of $\Omega$-figures. Despite eliciting robust granule fusion and catecholamine release, $15 \mathrm{~Hz}$ stimulation did not increase capacitance variance above unstimulated control values, indicative of full-collapse exocytosis (Fig. 5b). Cytochalasin $\mathrm{D}$ treatment and stimulation at $0.5 \mathrm{~Hz}$ resulted in low capacitance variance, indicating fusion pore dilation to drive fullcollapse exocytosis while having no effect under $15 \mathrm{~Hz}$ stimulation, where variance is low in control conditions (supplemental Table S2, available at www.jneurosci.org as supplemental material). Conversely, jasplakinolide treatment kept variance at an elevated level even under $15 \mathrm{~Hz}$ stimulation, which normally results in low variance, but did not affect variance under conditions that normally exhibit high values $(0.5 \mathrm{~Hz}$ stimulation) (supplemental Table S2, available at www.jneurosci.org as supplemental material). This result indicates that jasplakinolide blocks the transition from kiss-and-run exocytosis to full granule collapse that normally accompanies increased cell firing. These data are consistent with results obtained with the independent amperometric technique (Fig. 3) and indicate that at $0.5 \mathrm{~Hz}$ stimulation, filamentous actin plays a role in maintaining the restricted fusion pore associated with $\Omega$-form exocytosis. Disruption of filamentous actin affects the transition to full-collapse exocytosis.

Next, we tested the role of myosin II under $15 \mathrm{~Hz}$ stimulation. Treatment with the MLCK inhibitors ML7 or M18, or myosin II inhibitor blebbistatin, maintained high capacitance variance, indicating a persistence of kiss-and-run exocytosis (Fig. 5b). Furthermore, cotreatment with both jasplakinolide and M18, to stabilize actin and inhibit MLCK, did not produce an additive effect (Fig. 5b); measured variance was not greater than that measured in cells treated with jasplakinolide or M18 separately. This result indicates that jasplakinolide and M18 share a common mode of action, linking the role of actin and myosin II in the regulation of fusion pore dilation. Furthermore, none of these reagents altered variance under $0.5 \mathrm{~Hz}$ stimulation (see supplemental Table S2, available at www.jneurosci.org as supplemental material), indicating that MLCK and myosin II play a role only under stimulation conditions leading to full granule collapse.

\section{Endocytic uptake of fluorescent dextran is dependent on actin and myosin II}

We further tested the hypothesis that filamentous actin stabilizes a restricted fusion pore under kiss-and-run exocytosis. We used a fluorescence-based approach to probe for the diameter of the fusion/fission pore between cell membrane and newly forming endosomes under conditions with intact versus disrupted actin cortex. Seventy kilodalton dextran $(1 \mu \mathrm{M})$ has a molecular diam- 
eter of $\sim 12 \mathrm{~nm}$ (Ioan et al., 2000) and has been shown to be excluded from endosomes as they pinch from the cell surface under kiss-and-run exocytosis in both chromaffin cells and pancreatic $\beta$ cells (Takahashi et al., 2002; Fulop et al., 2005). Under $0.5 \mathrm{~Hz}$ stimulation, despite significant exocytic catecholamine release (Fig. 1), cells exhibited no accumulated intracellular fluorescence [Fig. $6 a$ (left image), bi $(\bigcirc)$ ], presumably because of exclusion of dextran uptake by the restricted fusion pore of the $\Omega$-figure. However, stimulation at the same $0.5 \mathrm{~Hz}$ frequency in cytochalasin D-treated cells resulted in significant internalization of dextran signal (Fig. 6bi, ). This result was not because of an enhanced secretory activity under cytochalasin D treatment; integrated amperometric currents showed that there was no difference in total catecholamine secretion after cytochalasin D treatment (assayed as total integrated amperometric current measured during the stimulation trains; control, $354 \pm 18 \mathrm{pC}$; cytochalasin D, $353 \pm 29 \mathrm{pC}$ ). Thus disruption of filamentous actin led to dilation of the fusion pore to a size greater than the diameter of $70 \mathrm{kDa}$ dextran. Conversely, stimulation at $15 \mathrm{~Hz}$ resulted in a robust dextran fluorescence uptake [Fig. $6 a$ (right image), bii $(\bigcirc)$ ]. Stabilization of filamentous actin (Fig. 6b, Jas, -) or blocking MLCK activity (Fig. 6b, ML7, $\bullet$ ) blocked dextran uptake under $15 \mathrm{~Hz}$ stimulation, despite levels of catecholamine release identical to that seen in control cells (assayed as above; control, $365 \pm 19$ pC; jasplakinolide, $366 \pm 30$ pC; ML7, $366 \pm$ $39 \mathrm{pC})$.

Together, the data presented here demonstrate that activitydependent disruption of the filamentous actin cortex and activation of myosin II play a critical role in the conversion from kissand-run to full-collapse exocytosis in adrenal chromaffin cells (Fig. 6c).

\section{Discussion}

Previous evidence demonstrated a correlation between stimulation and actin cytoskeleton dynamics in chromaffin cells. Bath application of potent chemical secretagogues established a stimulation-dependent dissolution of the dense actin cortex, concluding that this disruption was necessary for the sustained recruitment of granules from the cell interior to the membrane, where fusion and transmitter release occur (Nakata and Hirokawa, 1992; Trifaró et al., 1992; Vitale et al., 1995; Giner et al., 2005). Our data allow for a refinement of this interpretation. Using physiological stimulation designed to mimic basal electrical splanchnic firing under sympathetic tone (action potential equivalent waveforms delivered at $0.5 \mathrm{~Hz}$ ), we show that sustained catecholamine release occurs without significant gross reorganization of the actin cortex. Previous work from our lab has shown that these stimulation conditions correlate to specific catecholamine release but retention of the neuropeptide-containing granule core (Fulop et al., 2005). Amperometric, capacitance variance, and dye uptake data presented here (Figs. 1, 5, 6) collectively support the conclusion that exocytosis under lowfrequency stimulation conditions occurs through an $\Omega$-form fusion transient. Thus, specific catecholamine release under lowfrequency stimulation occurs through kiss-and-run exocytosis with an intact actin cortex and inactive myosin II. Stimulation mimicking splanchnic firing under the sympathetic stress response (action potential waveforms delivered at $15 \mathrm{~Hz}$ ) is then consistent with previous studies and shows disruption of the actin cortex and elevated catecholamine output. Again, collective data presented here obtained from amperometric spike analysis, capacitance variance measurements, and large fluid-phase dye uptake show that exocytosis under $15 \mathrm{~Hz}$ stimulation occurs pre-

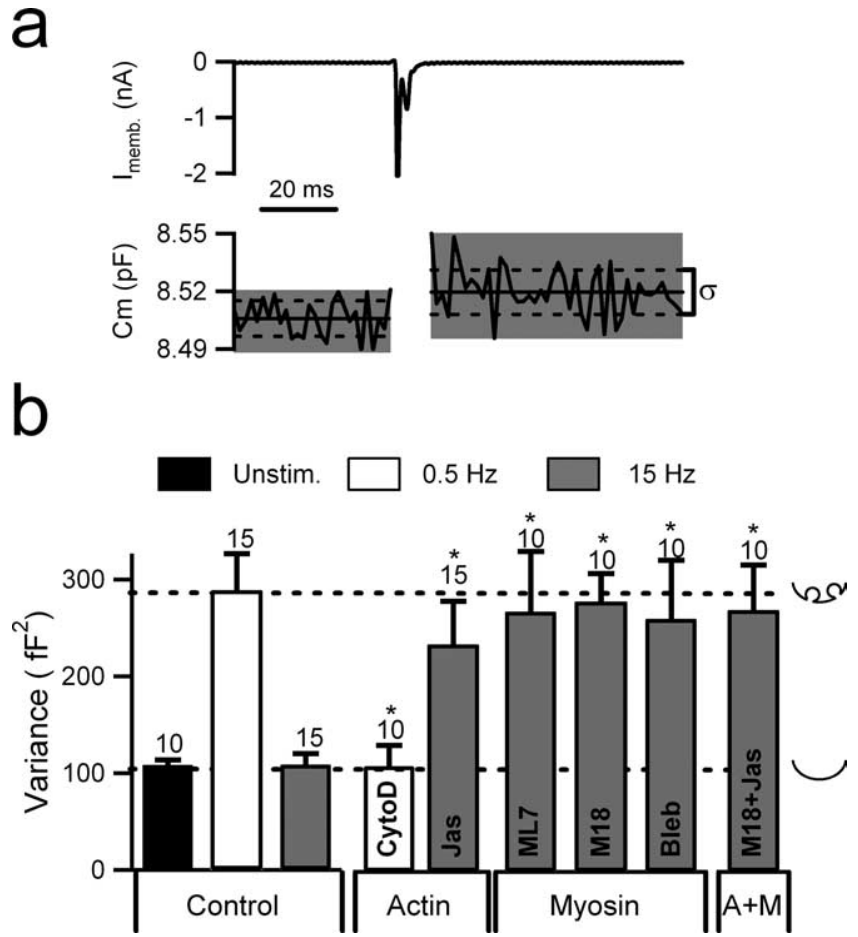

Figure 5. Variance analysis predicts actin and myosin II as regulators of kiss-and-run exocytosis. Capacitance variance was used to probe the roles of actin and myosin II in regulation of the $\Omega$-figure. $a$, Cells held in the perforated-patch configuration were stimulated with 0.5 or $15 \mathrm{~Hz}$ APe. Evoked membrane current (top) and measured cell capacitance (bottom) are shown for an example single APe stimulus. In response to $\mathrm{Ca}^{2+}$ influx, cell capacitance increases by $\sim 15 \mathrm{fF}$, indicating the fusion of eight or nine granules with the cell surface. Solid lines indicate linear fits to prepulse and postpulse capacitance. Dotted lines indicate the standard deviation $(\sigma)$ of these segments, and variance $\left(\sigma^{2}\right)$ is calculated after each stimulation. $\boldsymbol{b}$, Variance was calculated throughout stimulus trains of APe delivered at $0.5 \mathrm{~Hz}$ or $15 \mathrm{~Hz}$ in control and cells treated with agents that perturb filamentous actin, MLCK, or myosin II function. Concentrations for each agent were as in previous figures. Data are grouped according to pharmacological perturbation. Numbers of cells in each condition are indicated numerically above each category. Statistical significance was determined by Student's $t$ test with a significance barrier $(p)$ of 0.05 . Error bars represent SEM. Significance with respect to the untreated control group at the same stimulus frequency is indicated as an asterisk. For comparison across conditions, dotted lines are drawn to indicate the control values for 0.5 and $15 \mathrm{~Hz}$ stimulation. Icons to the right of the plot indicate the mode of fusion at each control variance level.

dominantly through a full-collapse exocytic mode. This shift in exocytic mode is associated with an activity-dependent disruption of the actin cortex (Fig. 2). Indeed, pharmacological perturbation of the actin cortex establishes a causative relationship between cortex organization and the mode of exocytosis. Disruption of the F-actin cortex with cytochalasin D and stimulation at $0.5 \mathrm{~Hz}$ resulted in a shift away from kiss-and-run toward full-collapse exocytosis by all parameters measured (amperometric spike analysis, capacitance variance analysis, and fluid-phase dye uptake). Conversely jasplakinolide, an F-actin stabilizer, had the opposite result. Under $15 \mathrm{~Hz}$ stimulation, which normally results in full-collapse exocytosis, jasplakinolide pretreatment favored maintenance of kiss-and-run exocytosis. An immediate interpretation of these data is that the actin cortex acts as a support scaffold to stabilize the $\Omega$-figure fusion transient in kiss-andrun exocytosis. Disruption of F-actin favors the collapse of the $\Omega$-figure intermediate to transition to full collapse.

We further show that myosin II also plays a complementary role in this process. Previous studies have shown that different myosin isoforms play an important role in regulating exocytosis (Trifaró et al., 2007). Indeed, chemical stimulation causes the 


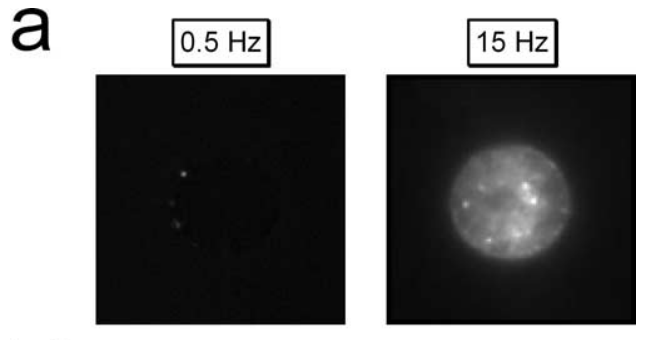

bi

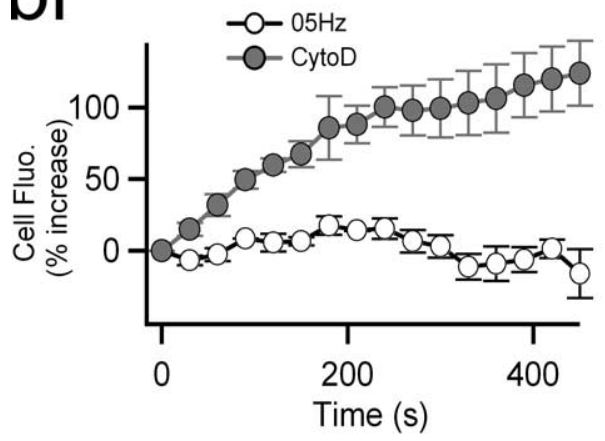

il

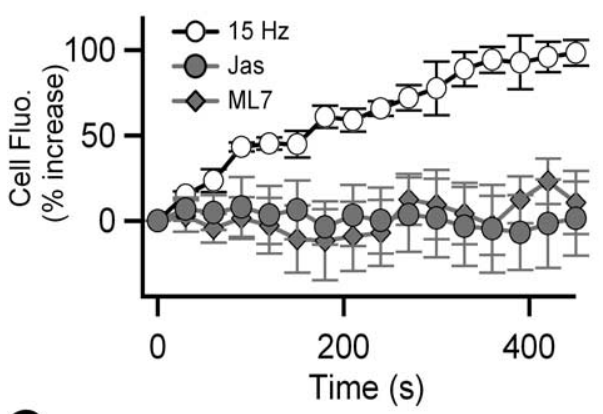

C

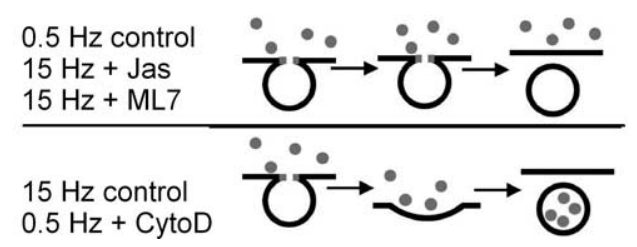

Figure 6. Dextran uptake indicates that actin and myosin II regulate fusion pore dilation. Chromaffin cells were stimulated with 0.5 or $15 \mathrm{~Hz}$ action potential waveforms, and Texas Red-conjugated fluorescent $70 \mathrm{kDa}$ dextran $(1 \mu \mathrm{m})$ was included in the bath solution as a fluid-phase marker. Cells were imaged during the stimulus train to probe for endosomal marker uptake. $\boldsymbol{a}$, Representative images show dye loading for each stimulus condition. $\boldsymbol{b i}$, Control cells stimulated at $0.5 \mathrm{~Hz}$ showed no $70 \mathrm{kDa}$ dextran uptake ( $n=8$ cells from 4 preparations). Pretreatment with $2 \mu \mathrm{m}$ cytochalasin D resulted in dextran uptake during stimulation $(n=7$ cells from 3 preparations). bii, Control cells stimulated at $15 \mathrm{~Hz}$ showed strong uptake of $70 \mathrm{kDa}$ dextran, whereas agents that stabilize actin (Jas) or inhibit MLCK (ML7) each blocked $70 \mathrm{kDa}$ dextran uptake $[n$ (cells/preparations) $=8 / 4,7 / 3$, and 9/3, respectively]. $c$, A diagram of the hypothesized modes of exocytosis under different stimulation conditions is pictured. Stimulation at $0.5 \mathrm{~Hz}$ or stabilization of F-actin results in kiss-and-run exocytosis, whereas $15 \mathrm{~Hz}$ stimulation or F-actin disruption leads to full collapse.

regulatory subunit of MLCK to become phosphorylated in a calcium-dependent manner proportional to the magnitude of the stimulus (Nicotera et al., 1986; Gutierrez et al., 1989). Furthermore, granule recruitment to the readily releasable pool has been shown to require phosphorylation of MLCK and activation of myosin (Kumakura et al., 1994; Rosé et al., 2002). Recent work found that expression of a phosphorylation-incompetent form of myosin II regulatory light chain decreased the kinetics of catecholamine release from single granules. These data demonstrate that myosin II both is required for vesicle mobility and may have a role in the later stages of exocytosis on the single-granule scale (Neco et al., 2002, 2004). Our data are consistent with, and build on, these data. We find that pharmacological perturbation of MLCK either by pretreatment with ML7 or by peptide transfection with M18 maintains kiss-and-run exocytosis in conditions that normally favor full collapse. Furthermore, direct inhibition of myosin II with blebbistatin mimicked this result, blocking the activity-dependent shift from kiss-and-run to full-collapse exocytosis. Cotreatment of cells with M18 to block MLCK and jasplakinolide to stabilize actin resulted in a complete transition between modes of exocytosis by all parameters measured. That the effects of M18 and jasplakinolide were not additive, and that they resulted quantitatively in a full conversion from full collapse to kiss-and-run under $15 \mathrm{~Hz}$ APe stimulation, indicates that they likely act cooperatively to regulate the mode of exocytosis.

Clearly, the cellular mechanism responsible for the activitydependent shift in the mode of exocytosis does not end with actin and myosin II. Previous work has demonstrated a specific role for stimulus-dependent $\mathrm{Ca}^{2+}$ elevations in the regulation of actin cortex (Trifaró et al., 2000, 2002). Increased $\mathrm{Ca}^{2+}$ activates the actin regulatory protein scinderin. Calcium-bound scinderin then severs F-actin to disrupt the actin cortex (Rodriguez Del Castillo et al., 1990). Similarly, a second pathway may translate increased cell firing into disruption of the actin cortex. In the unphosphorylated form, myristoylated alanine-rich C-kinase substrate (MARCKS) is bound to F-actin and stabilizes it in the polymerized form. Increased cell firing, and thus increased cytosolic $\mathrm{Ca}^{2+}$, may activate conventional isoforms of protein kinase $\mathrm{C}$ (PKC) to phosphorylate MARCKS. In the phosphorylated form, MARCKS dissociates from F-actin to favor actin depolymerization (Cuchillo-Ibáñez et al., 2004). Indeed, previous work has shown that cytosolic calcium levels under $0.5 \mathrm{~Hz}$ APe stimulation are insufficient to activate $\mathrm{PKC}$, whereas $15 \mathrm{~Hz}$ APe stimulation does activate PKC. PKC activation correlates with a shift in exocytic mode from kiss-and-run to full collapse. Moreover, pharmacological block or activation of PKC shifts the mode of exocytosis independent of stimulation intensity (Fulop and Smith, 2006). These potential regulatory paths may begin to outline a cellular signaling cascade whereby elevated cell firing leads to a $\mathrm{Ca}^{2+}$-mediated activation of scinderin and PKC. PKC then could phospho-activate MARCKS and cause it to act in concert with scinderin to dissociate the actin cortex, thus allowing the transition from kiss-and-run to full-collapse exocytosis. Activated PKC could then potentially phosphorylate MLCK (Clement et al., 1992; Vitale et al., 1995) to initiate the myosin IImediated contribution to the conversion in exocytotic mode. Further biophysical and biochemical experiments will be needed to test this potential pathway and to determine whether it represents the cellular events responsible for the transition between the sympathetic "breed and feed" and the "fight or flight" metabolic status.

\section{References}

Albillos A, Dernick G, Horstmann H, Almers W, Alvarez de Toledo G, Lindau M (1997) The exocytotic event in chromaffin cells revealed by patch amperometry. Nature 389:509-512.

Alvarez de Toledo G, Fernández-Chacón R, Fernández JM (1993) Release of secretory products during transient vesicle fusion. Nature 363:554-558.

Aravanis AM, Pyle JL, Tsien RW (2003) Single synaptic vesicles fusing transiently and successively without loss of identity. Nature 423:643-647. 
Artalejo CR, Elhamdani A, Palfrey HC (2002) Sustained stimulation shifts the mechanism of endocytosis from dynamin-1-dependent rapid endocytosis to clathrin- and dynamin-2-mediated slow endocytosis in chromaffin cells. Proc Natl Acad Sci USA 99:6358-6363.

Barclay JW, Craig TJ, Fisher RJ, Ciufo LF, Evans GJ, Morgan A, Burgoyne RD (2003) Phosphorylation of Munc18 by protein kinase C regulates the kinetics of exocytosis. J Biol Chem 278:10538-10545.

Brandt B, Hagiwara S, Kidokoro Y, Miyazaki S (1976) Action potentials in the rat chromaffin cell and effects of acetylcholine. J Physiol (Lond) 263:417-439.

Chan SA, Smith C (2001) Physiological stimuli evoke two forms of endocytosis in bovine chromaffin cells. J Physiol (London) 537:871-885.

Chen P, Gillis KD (2000) The noise of membrane capacitance measurements in the whole-cell recording configuration. Biophys J 79:2162-2170.

Chow RH, von Ruden L, Neher E (1992) Delay in vesicle fusion revealed by electrochemical monitoring of single secretory events in adrenal chromaffin cells. Nature 356:60-63.

Clement O, Puceat M, Walsh MP, Vassort G (1992) Protein kinase C enhances myosin light-chain kinase effects on force development and ATPase activity in rat single skinned cardiac cells. Biochem J 285:311-317.

Cuchillo-Ibáñez I, Lejen T, Albillos A, Rosé SD, Olivares R, Villarroya M, García AG, Trifaró J-M (2004) Mitochondrial calcium sequestration and protein kinase $\mathrm{C}$ cooperate in the regulation of cortical F-actin disassembly and secretion in bovine chromaffin cells. J Physiol (Lond) 560:63-76.

Elhamdani A, Palfrey HC, Artalejo CR (2001) Quantal size is dependent on stimulation frequency and calcium entry in calf chromaffin cells. Neuron 31:819-830.

Elhamdani A, Azizi F, Artalejo CR (2006) Double patch clamp reveals that transient fusion (kiss-and-run) is a major mechanism of secretion in calf adrenal chromaffin cells: high calcium shifts the mechanism from kissand-run to complete fusion. J Neurosci 26:3030-3036.

Fulop T, Smith C (2006) Physiological stimulation regulates the exocytic mode through calcium activation of protein kinase $\mathrm{C}$ in mouse chromaffin cells. Biochem J 399:111-119.

Fulop T, Smith C (2007) Matching native electrical stimulation by graded chemical stimulation in isolated mouse adrenal chromaffin cells. J Neurosci Methods 166:195-202.

Fulop T, Radabaugh S, Smith C (2005) Activity-dependent differential transmitter release in mouse adrenal chromaffin cells. J Neurosci 25:7324-7332.

Gillis KD (1995) Chapter 7. Techniques for membrane capacitance measurements. In: Single-channel recording, Ed 2 (Sakmann B, Neher E, eds), pp 155-198. New York: Plenum.

Giner D, Neco P, del Mar Francés M, López I, Viniegra S, Gutiérrez LM (2005) Real-time dynamics of the F-actin cytoskeleton during secretion from chromaffin cells. J Cell Sci 118:2871-2880.

Giner D, López I, Villanueva J, Torres V, Viniegra S, Gutiérrez LM (2007) Vesicle movements are governed by the size and dynamics of F-actin cytoskeletal structures in bovine chromaffin cells. Neuroscience $146: 659-669$.

Gomez JF, Brioso MA, Machado JD, Sanchez JL, Borges R (2002) New approaches for analysis of amperometrical recordings. Ann N Y Acad Sci 971:647-654.

Gutierrez LM, Hidalgo MJ, Palmero M, Ballesta JJ, Reig JA, Garcia AG, Viniegra S (1989) Phosphorylation of myosin light chain from adrenomedullary chromaffin cells in culture. Biochem J 264:589-596.

Habib KE, Gold PW, Chrousos GP (2001) Neuroendocrinology of stress. Endocrinol Metab Clin North Am 30:695-728; vii-viii.

Ioan C, Aberle T, Burchard W (2000) Structure properties of dextran. 2. Dilute solution. Macromolecules 33:5730-5739.

Kidokoro Y, Ritchie AK (1980) Chromaffin cell action potentials and their possible role in adrenaline secretion from rat adrenal medulla. J Physiol (Lond) 307:199-216.

Korn SJ, Horn R (1989) Influence of sodium-calcium exchange on calcium current rundown and the duration of calcium-dependent chloride currents in pituitary cells, studied with whole cell and perforated patch recording. J Gen Physiol 94:789-812.

Kumakura K, Sasaki K, Sakurai T, Ohara-Imaizumi M, Misonou H, Nakamura S, Matsuda Y, Nonomura Y (1994) Essential role of myosin light chain kinase in the mechanism for MgATP-dependent priming of exocytosis in adrenal chromaffin cells. J Neurosci 14:7695-7703.

Lindau M, Alvarez de Toledo G (2003) The fusion pore. Biochim Biophys Acta 1641:167-173.

Lukas TJ, Mirzoeva S, Slomczynska U, Watterson DM (1999) Identification of novel classes of protein kinase inhibitors using combinatorial peptide chemistry based on functional genomics knowledge. J Med Chem 42:910-919.

Nakata T, Hirokawa N (1992) Organization of cortical cytoskeleton of cultured chromaffin cells and involvement in secretion as revealed by quickfreeze, deep-etching, and double-label immunoelectron microscopy. J Neurosci 12:2186-2197.

Neco P, Gil A, Del Mar Francés M, Viniegra S, Gutiérrez LM (2002) The role of myosin in vesicle transport during bovine chromaffin cell secretion. Biochem J 368:405-413.

Neco P, Giner D, Viniegra S, Borges R, Villarroel A, Gutiérrez LM (2004) New roles of myosin II during vesicle transport and fusion in chromaffin cells. J Biol Chem 279:27450-27457.

Neher E, Marty A (1982) Discrete changes of cell membrane capacitance observed under conditions of enhanced secretion in bovine adrenal chromaffin cells. Proc Natl Acad Sci USA 79:6712-6716.

Nicotera P, Hartzell P, Davis G, Orrenius S (1986) The formation of plasma membrane blebs in hepatocytes exposed to agents that increase cytosolic $\mathrm{Ca} 2+$ is mediated by the. FEBS Lett 209:139-144.

Rahamimoff R, Fernandez JM (1997) Pre- and postfusion regulation of transmitter release. Neuron 18:17-27.

Richards DA, Bai J, Chapman ER (2005) Two modes of exocytosis at hippocampal synapses revealed by rate of FM1-43 efflux from individua vesicles. J Cell Biol 168:929-939.

Rodriguez Del Castillo A, Lemaire S, Tchakarov L, Jeyapragasan M, Doucet JP, Vitale ML, Trifaró J-M (1990) Chromaffin cell scinderin, a novel calcium-dependent actin filament-severing protein. EMBO J 9:43-52.

Rosé SD, Lejen T, Casaletti L, Larson RE, Pene TD, Trifaró J-M (2002) Molecular motors involved in chromaffin cell secretion. Ann N Y Acad Sci 971:222-231.

Saitoh M, Ishikawa T, Matsushima S, Naka M, Hidaka H (1987) Selective inhibition of catalytic activity of smooth muscle myosin light chain kinase. J Biol Chem 262:7796-7801.

Takahashi N, Kishimoto T, Nemoto T, Kadowaki T, Kasai H (2002) Fusion pore dynamics and insulin granule exocytosis in the pancreatic islet. Science 297:1349-1352.

Trifaró J-M, Vitale ML, Rodriguez DCA (1992) Cytoskeleton and molecular mechanisms in neurotransmitter release by neurosecretory cells. Eur J Pharmacol 225:83-104.

Trifaró J-M, Rosé SD, Lejen T, Elzagallaai A (2000) Two pathways control chromaffin cell cortical F-actin dynamics during exocytosis. Biochimie 82:339-352.

Trifaró J-M, Lejen T, Rosé SD, Pene TD, Barkar ND, Seward EP (2002) Pathways that control cortical F-actin dynamics during secretion. Neurochem Res 27:1371-1385.

Trifaró J-M, Gasman S, Gutiérrez LM (2007) Cytoskeletal control of vesicle transport and exocytosis in chromaffin cells. Acta Physiol (Oxf) 192:165-172.

Vitale ML, Rodriguez Del Castillo A, Tchakarov L, Trifaró J-M (1991) Cortical filamentous actin disassembly and scinderin redistribution during chromaffin cell stimulation precede exocytosis, a phenomenon not exhibited by gelsolin. J Cell Biol 113:1057-1067.

Vitale ML, Seward EP, Trifaró J-M (1995) Chromaffin cell cortical actin network dynamics control the size of the release-ready vesicle pool and the initial rate of exocytosis. Neuron 14:353-363.

Wieland T (1986) Peptides of poisonous amanita mushrooms, Ed 1. New York: Springer.

Wightman RM, Jankowski JA, Kennedy RT, Kawagoe KT, Schroeder TJ, Leszczyszyn DJ, Near JA, Diliberto EJ, Viveros OH (1991) Temporally resolved catecholamine spikes correspond to single vesicle release from individual chromaffin cells. Proc Natl Acad Sci USA 88:10754-10758.

Wilson JM, Coderre E, Renaud LP, Spanswick D (2002) Active and passive membrane properties of rat sympathetic preganglionic neurones innervating the adrenal medulla. J Physiol (Lond) 545:945-960. 